Industrial grade versus scientific pure: Influence on melt properties

I. Jonas, W. Hembree, F. Yang, R. Busch, and A. Meyer

Citation: Appl. Phys. Lett. 112, 171902 (2018); doi: 10.1063/1.5021764

View online: https://doi.org/10.1063/1.5021764

View Table of Contents: http://aip.scitation.org/toc/apl/112/17

Published by the American Institute of Physics

MANAGER'S GUIDE

\section{PHYSICS TODAY}

WHITEPAPERS

Accelerate R\&D with

Multiphysics Simulation
READ NOW

PRESENTED BY

$\checkmark \subset \mathrm{MSOL}$ 


\title{
Industrial grade versus scientific pure: Influence on melt properties
}

\author{
I. Jonas, ${ }^{1, a)}$ W. Hembree ${ }^{2}$ F. Yang, ${ }^{1}$ R. Busch, ${ }^{2}$ and A. Meyer ${ }^{1}$ \\ ${ }_{1}^{1}$ Institut für Materialphysik im Weltraum, Deutsches Zentrum für Luft-und Raumfahrt (DLR), 55170 Köln, \\ Germany \\ ${ }^{2}$ Chair of Metallic Materials, Saarland University, 66123 Saarbrücken, Germany
}

(Received 8 January 2018; accepted 10 April 2018; published online 24 April 2018)

\begin{abstract}
Viscosity, density, and the undercooling ability of the Zr-based bulk glass forming melt, which was manufactured in two different degrees of purity, have been studied. Investigations have been carried out by means of Couette rheometry and electrostatic and electromagnetic levitation with the latter under microgravity conditions. We found that oxygen and impurities present in industrial grade metals do not significantly alter the melt viscosity and density, while they clearly affect the undercooling ability. Comparing container based and containerless results showed that Couette rheometry can be applied in the temperature range between $1150 \mathrm{~K}$ and $1375 \mathrm{~K}$, where it provides reliable data, but only at a rather low oxygen content. Higher oxygen contents, as in the case of the industrial grade alloy, cause measurement artefacts. In the case of $\mathrm{Zr}_{59.3} \mathrm{Cu}_{28.8} \mathrm{Al}_{10.4} \mathrm{Nb}_{1.5}$ alloys, these findings allow a better localization of the key factors dominating the glass forming ability. Published by AIP Publishing. https://doi.org/10.1063/1.5021764
\end{abstract}

Fundamental research on bulk metallic glasses (BMGs) emanates mostly from high purity starting materials. However, the purity of the starting materials is an important cost driver for applications. Industrial grade metals are usually considerably inexpensive but contain substantially more impurities, especially oxygen. ${ }^{1,2}$ For industrial applications of BMGs, it is thus important to find out whether using starting materials containing a higher amount of impurities affects the properties of the melt and thus influences the production process.

Here, we studied two different degrees of purity of the $\mathrm{Zr}$ based bulk metallic glass AMZ4. High purity AMZ4 $\left(\mathrm{Zr}_{59.3} \mathrm{Cu}_{28.8} \mathrm{Al}_{10.4} \mathrm{Nb}_{1.5}\right)$ was processed from $99.9 \%$ to $99.99 \%$ pure metals. Industrial AMZ4 $\left(\mathrm{Zr} 705_{60.8} \mathrm{Cu}_{20.8} \mathrm{Al}_{10.4}\right)$ replaces the high purity Zirconium and Niobium with the industrial grade pre-alloy Zr R60705., This alloy is composed of up to 95.5\% Zirconium and Hafnium with a maximum Hafnium content of $4.5 \%$. In addition, it contains $2 \%-3 \%$ Niobium and approximately $1 \%$ Oxygen. ${ }^{4}$ Further details are given in Table I. The manufacturing costs for these two grades differ by a factor of $9.5^{4-6}$

In the following, the influence of impurities, and in particular that of oxygen in starting materials of the AMZ4 melt, is investigated. This is achieved by measurements of viscosity, density, and the undercooling ability. The effects on the glass forming ability (GFA) are discussed.

Viscosities were measured in the equilibrium and undercooled melt by Couette rheometry and electrostatic and electromagnetic levitation (ESL ${ }^{7}$ and $\mathrm{EML}$ ) experiments in combination with the oscillating drop technique. ${ }^{8,9}$ EML measurements were conducted under reduced gravity $(\mu \mathrm{g})$ conditions in the TEMPUS facility (Tiegelfreies Elektromagnetisches Prozessieren Unter Schwerelosigkeit). The ESL technique was also used to study density ${ }^{10,11}$ and undercooling ability of the alloy melts.

Master alloys of industrial and high purity AMZ4 were prepared by arc-melting of elemental metals with respective

a)isabell.jonas@dlr.de purities (see Table I). These ingots were cast into amorphous samples by subsequent re-melting and pressure assisted gravity casting into water cooled copper molds of appropriate geometry. In order to eliminate any influence from the sample material on the different measurements, samples under investigation were prepared from the same production batch. ${ }^{12}$

The rheometer used is a custom built rotating concentric cylinder (Couette) rheometer, which can be operated at controlled shear rates and temperatures. The tapered bob of this conicylinder shear cell type prevents the entrapment of bubbles that may otherwise disturb measurements. Further information is given in Ref. 12. The temperature dependent flow behavior was investigated by first heating the samples to $300 \mathrm{~K}$ above $\mathrm{T}_{\text {liq }}$ and then applying a stepwise shear rate profile in both clockwise and counter clockwise directions. While shearing, the melt was cooled down to near the melting temperature at a rate of $0.33 \mathrm{~K} \mathrm{~s}^{-1}$ and then immediately reheated to the starting temperature at the same rate. This cooling and heating cycle was repeated a total of three times for different shear rates of $228 \mathrm{~s}^{-1}, 342 \mathrm{~s}^{-1}$, and $456 \mathrm{~s}^{-1}$. Since no systematic difference in viscosity was observed for the different shear rates, only data measured at $456 \mathrm{~s}^{-1}$ are displayed. The volume of the graphite Couette cup is $6 \mathrm{~cm}^{3}$, which corresponds to a sample mass of some $40 \mathrm{~g}$.

Samples used in ESL weighed between 40 and $90 \mathrm{mg}$ and were processed under high vacuum $\left(<10^{-7} \mathrm{mbar}\right)$.

TABLE I. Manufacturer's specifications for Zirconium grades as used in high purity AMZ4 made of purified Zirconium ${ }^{6}$ and industrial AMZ4 made of Zr R60705. ${ }^{4}$

\begin{tabular}{lcc}
\hline \hline Element & Purified $\mathrm{Zr}^{6}$ & $\mathrm{Zr} \mathrm{R60705^{4 }}$ \\
\hline Oxygen $(\mathrm{ppm})$ & 70 & $10300(\approx 1$ at. $\%)$ \\
Hydrogen $(\mathrm{ppm})$ & 25 & 4540 \\
Nitrogen $(\mathrm{ppm})$ & 20 & 1630 \\
Carbon $(\mathrm{ppm})$ & $\mathrm{n} / \mathrm{a}$ & $3810 \mathrm{ppm}$ \\
Iron and chromium $(\mathrm{ppm})$ & 300 & 3280 \\
Niobium $(\mathrm{ppm})$ & 50 & $30000(\approx 3$ at. $\%)$ \\
\hline \hline
\end{tabular}


Heating or melting of samples was achieved using a $25 \mathrm{~W}$ $(810 \mathrm{~nm})$ diode laser. The sample temperature was monitored using a single color pyrometer $(1450-1800 \mathrm{~nm})$ covering temperatures from $300^{\circ} \mathrm{C}$ to $2000^{\circ} \mathrm{C}$. A temperature correction was carried out via a reference temperature assuming a constant emissivity of the sample in the temperature region of interest. ${ }^{13}$ The reference temperature was determined from differential scanning experiments (DSC) at $10 \mathrm{~K} / \mathrm{min}$.

Melt viscosities were determined by means of induced surface oscillations initiated by an additional sinusoidal voltage superimposed on the levitation voltage. After stopping this induced oscillation, the damping time $\tau$ of the oscillation amplitude was determined. ${ }^{8}$ With this information, the viscosity $\eta$ can be calculated as

$$
\eta=\frac{\rho r_{0}^{2}}{\tau(l-1)(2 l+1)}
$$

where $\rho$ is the density, $r_{0}$ is the radius of the droplet when assuming a spherical shape, and $l$ is the oscillation mode, which in this work is $l=2$. Excitation frequencies are between $115 \mathrm{~Hz}$ and $155 \mathrm{~Hz}$ depending on the sample mass. Further information is given in Ref. 8. The results displayed here always include measurements with different sample masses, and no mass dependence was observed.

Viscosity measurements under reduced gravity conditions were performed in the TEMPUS facility on parabolic flights. ${ }^{14}$ Here, EML is combined with the oscillating drop method and viscosities are determined during $\approx 20 \mathrm{~s}$ of reduced gravity of a level of $\approx 0.01 \mathrm{~g}$. Samples possess a diameter of $6-8 \mathrm{~mm}$ and accordingly a weight of approximately $1.5 \mathrm{~g}$. In the TEMPUS facility, levitation and heating processes are decoupled by employing a two coil system. ${ }^{8}$ Surface oscillations are induced via a single rectangular pulse of a tenth of a second executed by the heating coil. The decay behavior was monitored with a camera system. Temperatures were tracked using a single color pyrometer. Analyses were carried out in the same way as in ESL experiments.

The results of Couette rheometer measurements are displayed in Fig. 1. These measurements cover a temperature range of around $200 \mathrm{~K}$ and a viscosity range of $20 \mathrm{mPa}$ s to $175 \mathrm{mPa}$ s. Industrial and high purity AMZ4 differ in absolute viscosity by a factor of approximately 2 but show similar temperature dependencies.

Viscosities measured using the ESL technique are displayed in Fig. 2. Here, ESL data cover a temperature range of around $400 \mathrm{~K}$ and a viscosity range from $15 \mathrm{mPa}$ s to 200 $\mathrm{mPa}$ s. Within the ESL results, only a small difference between the viscosity of industrial and high purity AMZ4 is found in contrast to Couette rheometer results. Couette and ESL data for high purity AMZ4 coincide with each other, as shown in Fig. 3 (bottom).

To further investigate the difference in industrial AMZ4 viscosity, the existing data are compared with viscosity data measured in benchmarking experiments performed in the TEMPUS facility. The results are displayed in Fig. 3 (top). The large temperature uncertainty of $\mu \mathrm{g}$ results is due to the fact that in the TEMPUS facility, viscosity measurements can only be conducted during free cooling. Every excitation

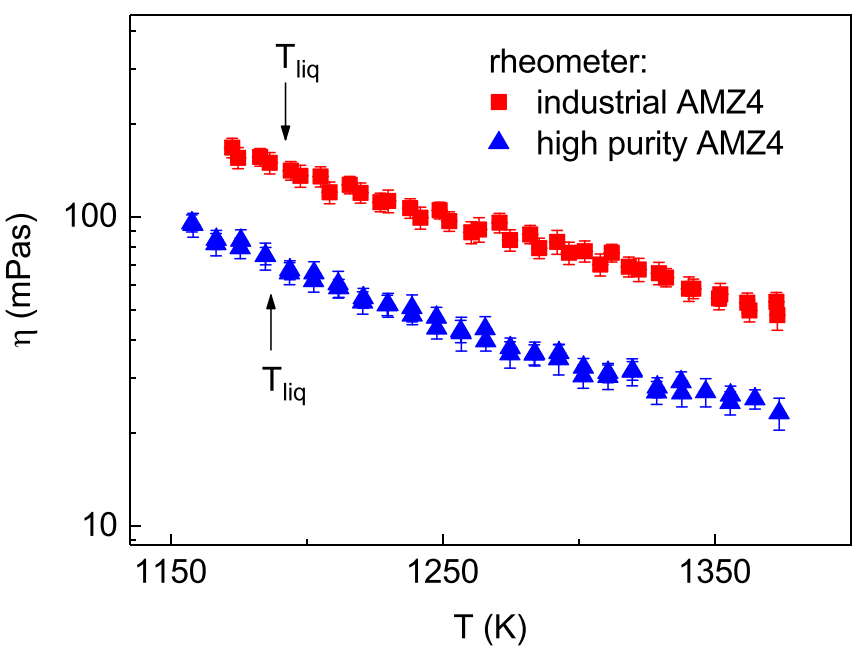

FIG. 1. Container-based Couette rheometer measurements of high purity and industrial AMZ4 upon cooling and heating. Liquidus temperatures $\left(\mathrm{T}_{\text {liq }}\right)$ are determined by means of DSC scans to be $1186 \mathrm{~K}$ and $1192 \mathrm{~K}$ for high purity and industrial AMZ4, respectively.

pulse is accompanied by a small increase in temperature and during the decay time of about $1 \mathrm{~s}$, the temperature can change up to $40 \mathrm{~K}$, depending on the starting temperature. The temperature of the viscosity displayed in Fig. 3 represents a mean temperature of the measured temperature range. The uncertainty in viscosity is attributed to the sample size, instability during the levitation process, and the estimated influence of the uncertainty in temperature $(\Delta T)$ on the viscosity. Nevertheless, it can be seen from Fig. 3 (top) that TEMPUS viscosities agree with ESL data within measurement uncertainties, while Couette data continue to deviate by a factor of approximately 2 .

It has been $\operatorname{shown}^{8}$ that ESL in combination with the oscillating drop method is an excellent tool for studying the viscosity of metallic glass forming melts in the range of about $10 \mathrm{mPas}$ to $250 \mathrm{mPas}$, with sample masses below $100 \mathrm{mg}$. Within ESL measurements of this work, no mass dependence was found (Fig. 2). Additional TEMPUS data give identical results [see Fig. 3 (top)]. This shows that the

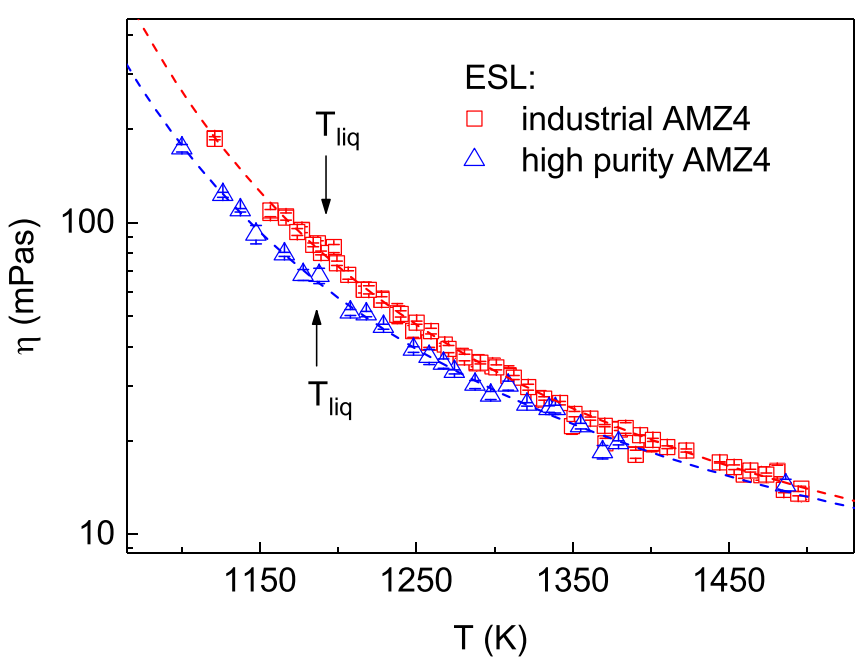

FIG. 2. Melt viscosities of high purity and industrial AMZ4 as determined by electrostatic levitation in combination with the oscillating drop technique. 


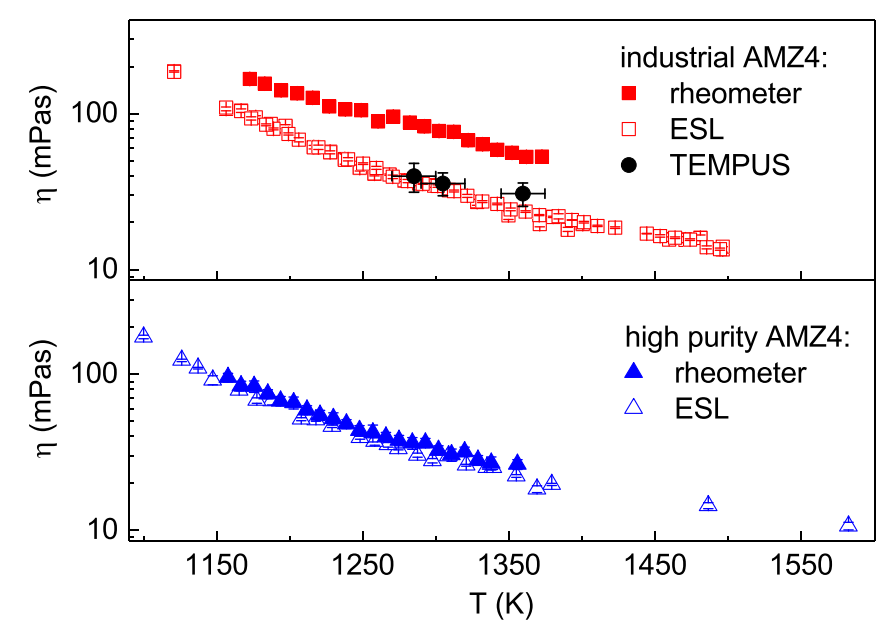

FIG. 3. Melt viscosities determined by Couette rheometry as well as by electrostatic and electromagnetic levitation in combination with the oscillating drop technique with the latter under reduced gravity conditions in the TEMPUS facility. Top: Data of industrial AMZ4. Bottom: Data of high purity AMZ4.

viscosity measured with the ESL technique experiences no external disturbances and is free of measurement artefacts.

Experimental viscosities determined by Couette rheometry crucially depend on interactions between the melt and the container material. It is known that if a graphite crucible is used, usually a stable zirconium carbide ( $\mathrm{ZrC}$ ) layer forms at the interface between the melt and the shear cell. ${ }^{15}$ This layer can remain stable at thicknesses below $10 \mu \mathrm{m}$ over the course of an $8 \mathrm{~h}$ long experiment ${ }^{12}$ and acts as a diffusion barrier $^{15,16}$ so that no further melt reactions take place and the sample composition remains chemically stable. It also ensures that the stick-slip boundary conditions for rheometer measurements are fulfilled. Thus, the following options are possible for causing the difference observed in the viscosity of industrial AMZ4 (Fig. 3): A reaction of the melt with the crucible material. This would lead to chemical changes of the melt composition. However, this reaction is time dependent. Measurements upon cooling and subsequent heating showed no difference in viscosity. Therefore, a chemical reaction is ruled out. Furthermore, the stability of the zirconium carbide layer might be affected by the presence of oxygen or other impurities and thus modify the diffusion behavior. Yet, oxygen and/or impurities might prevent the formation of this layer in the first place. Temperature and shear rate dependent behavior remained similar to that of high purity AMZ4 over the duration of the experiment. Therefore, one possible explanation is the contamination of the melt and the formation of slag on the surface already at the beginning of the experiment due to the use of impure $\mathrm{Zr}$ R60705. For high purity AMZ4, such a reaction appears to be less problematic since the alloy contains significantly less oxygen.

Our Couette rheometer experiments showed that measuring industrial grade metals with an oxygen content of 1 at. \% can lead to a supposed difference in viscosity by a factor of 2. ${ }^{8}$ Our ESL and EML experiments in contrast proved that the viscosity barely changes at an increased oxygen and/or impurity content. The significance of these findings is based on the fact that viscosity is an important material parameter, e.g., in casting processes or fragile-tostrong ${ }^{17}$ and liquid-liquid transitions. ${ }^{18}$

To further investigate material properties, which may affect the GFA, we studied the density as well as the undercooling ability of the alloys. Figure 4 (top) shows temperature dependent density for both AMZ4 alloys. The liquid density $\rho$ was found to decrease linearly with increasing temperature $\mathrm{T}$ as described by $\rho(T)=6.82\left(\mathrm{~g} / \mathrm{cm}^{3}\right)-3.48 \times 10^{-4} \mathrm{~T}\left(\mathrm{~g} / \mathrm{cm}^{3} \mathrm{~K}^{-1}\right)$. The liquid densities are almost identical for the two alloys. But high purity AMZ4 covers a larger temperature range from $850 \mathrm{~K}$ to $1575 \mathrm{~K}$, while industrial AMZ4 covers only a temperature range from $1080 \mathrm{~K}$ to $1500 \mathrm{~K}$. This is due to the fact that high purity AMZ4 can be undercooled to a much lower temperature in ESL experiments compared to industrial AMZ4. This is illustrated in Fig. 4 (bottom) in time-temperature profiles with the same amount of overheating for both alloys. High purity AMZ4 undercools approximately $350 \mathrm{~K}$ below its liquidus temperature of $\mathrm{T}_{\text {liq }}=1186 \mathrm{~K}$, while industrial AMZ4 undercools only about $125 \mathrm{~K}$ below its liquidus temperature of $\mathrm{T}_{l i q}=1192 \mathrm{~K}$.

It can be noted from Fig. 4 that for the high-purity AMZ4 alloy with a larger undercooling, there is almost no plateau in the time-temperature profile after the recalescence, whereas for the industrial grade alloy with a smaller undercooling, the temperature remained almost unchanged for approximately $15 \mathrm{~s}$ after the recalescence. This indicates that the high purity AMZ4 is solidified almost under fully nonequilibrium conditions, whilst for the industrial alloy at least parts of the melt solidify under equilibrium conditions after the first recalescence. ${ }^{19}$ Moreover, for the low purity alloy, spike-like features occur in the temperature profile after the recalescence. Since during equilibrium solidification the temperature is almost constant, they should be attributed to the emissivity change rather than the temperature change. Indeed, oxides on the sample surface, which have a higher emissivity than metals, could cause such spikes. However, grain boundaries or the coexistence of solid and liquid phases during the equilibrium solidification can also lead to such emissivity changes and spike-like features in the time-temperature profile. We hence cannot unambiguously assign this to oxides on the sample surface. However, this

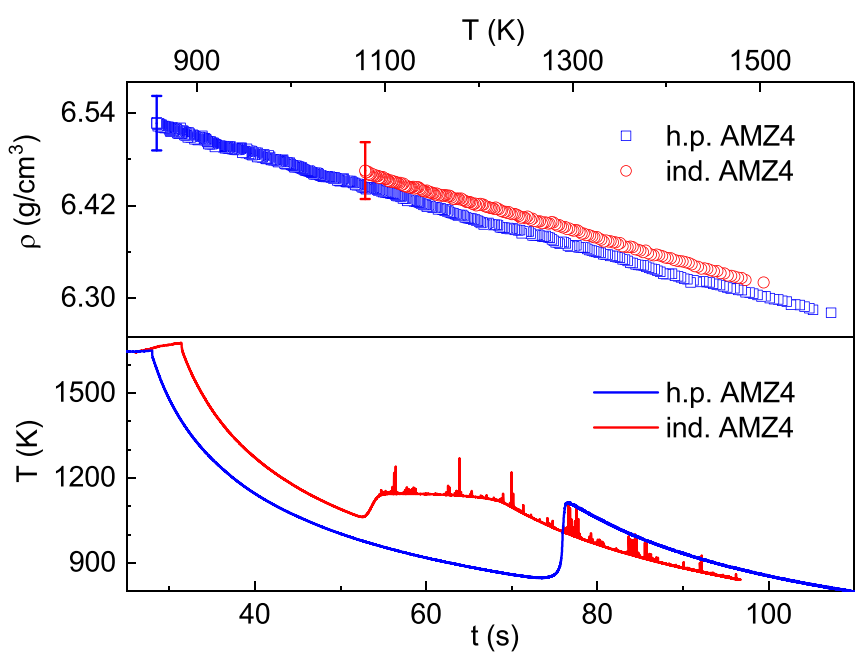

FIG. 4. Experimental data of industrial and high purity AMZ4. Top: Temperature dependent density course investigated with ESL. Bottom: Time-temperature profiles of ESL free radiation cooling experiments. 
shows that high purity and industrial grade alloys exhibit different solidification behaviors, due to different degrees of undercooling.

The undercooling ability is significantly diminished by an increased level of oxygen. Liu et al. ${ }^{20}$ found that the detrimental effect of oxygen in $\mathrm{Zr}$-based BMGs is due to the formation of oxygen-induced nuclei, which serve as nucleation sites for subsequent crystallization of the glass phase. This finding is in agreement with our experimental results.

In the literature, casting thickness $\left(d_{\max }\right)$, critical cooling rate $\left(R_{c r i t}\right)$, and time to bypass the nose in a time-temperature-transformation (TTT) diagram $\left(\tau_{X}\right)$ are interchangeably used as alternative measures of the GFA. ${ }^{21}$ We found the critical casting thicknesses as achieved within this work to be $5 \mathrm{~mm}$ for industrial AMZ4 and $12-14 \mathrm{~mm}$ for high purity AMZ4. Johnson et al. ${ }^{21}$ made an empirical approach for high GFA alloys, such as AMZ4 to set $\tau_{X}$ and $d_{\max }$ in relation via

$$
\tau_{X}^{*}(\mathrm{~s})=0.00419 d_{\max }(\mathrm{mm})^{2.54} .
$$

According to this relation, ${ }^{22}$ the critical time to bypass the nose in a TTT diagram is $0.25 \mathrm{~s}$ for industrial AMZ4 and $2.3 \mathrm{~s}$ to $3.4 \mathrm{~s}$ for high purity AMZ4. This is in line with our findings to such a degree that high purity AMZ4 undercools nearly three times as far. However, these nose times are still not sufficient to achieve complete vitrification. Lin and Johnson. ${ }^{23}$ established a relation between the critical cooling rate and the maximum casting thickness of an amorphous alloy. This relation reads

$$
R_{\text {crit }}(\mathrm{K} / \mathrm{s})=10 / d_{\max }(\mathrm{cm}) .
$$

Critical cooling rates are accordingly $40 \mathrm{~K} / \mathrm{s}$ for industrial AMZ4 and $5.1 \mathrm{~K} / \mathrm{s}-6.9 \mathrm{~K} / \mathrm{s}$ for high purity AMZ4.

Kinetic parameters such as the kinetic fragility enter many models predicting the glass forming ability. ${ }^{21,24}$ These parameters are typically determined from properties like viscosity. ${ }^{21}$ In our case of industrial grade and scientific pure AMZ4, it is unexpected ${ }^{25}$ that different glass forming abilities are found for the two alloys, whereas the equilibrium melt viscosities are almost identical. This becomes clear when looking at the kinetic fragility $D^{*}=9.6$ and the corresponding kinetic fragility index $m$ of 77.4 for high purity AMZ4. According to Ref. 21, this fragility index corresponds to a casting thickness of about $2.5 \mathrm{~cm}$, which agrees with experimental casting thicknesses within a factor of 2 . Accordingly, an almost identical casting thickness is predicted for industrial AMZ4, which is obviously not true. Thus, the different glass forming abilities cannot be taken into account by the model of Ref. 21. The reason for this is due to the fact that oxygen in undercooled $\mathrm{Zr}$-based melts induces heterogeneous nucleation, ${ }^{20}$ as shown by the undercooling ability of the melt, whereas in the model of Ref. 21, only homogeneous nucleation is assumed. In order to make predictions for industrial grade BMGs, the model would have to take heterogeneous nucleation into account.

In conclusion, we studied the melt properties viscosity, density, and the undercooling ability of the bulk metallic glass AMZ4, which was manufactured in two different degrees of purity by using Couette rheometry and electrostatic and electromagnetic levitation with the latter under microgravity conditions. Our findings show that oxygen, even at a level of 1 at. \%, does not significantly alter the melt density and viscosity, while it clearly diminishes the undercooling ability. The reason for the difference in glass forming ability is most likely due to the fact that oxygen in undercooled $\mathrm{Zr}$-based melts induces homogeneous nucleation, ${ }^{20}$ as indicated by the undercooling ability of the melt. This fact is not yet considered in models using kinetic parameters to predict the glass forming ability.

Furthermore, our measurements provide benchmarking criteria to verify results obtained by conventional measurement techniques. ${ }^{8,15}$ Container-based Couette rheometer measurements can provide reliable results up to $1375 \mathrm{~K}$ for Zr-based BMGs, if the oxygen content is not too high, in the purity range from $99.9 \%$ to $99.99 \%$ (see Table I).

The financial support provided by the Deutsche Forschungsgemeinschaft (DFG) through the Grant Nos. ME 1958/11-2 and BU 2276/6-2 is gratefully acknowledged.

${ }^{1}$ D. V. Louzguine-Luzgin, C. Suryanarayana, T. Saito, Q. Zhang, N. Chen, J. Saida, and A. Inoue, Intermetallics 18, 1531 (2010).

${ }^{2}$ X. H. Lin, W. L. Johnson, and W. K. Rhim, Mater. Trans., JIM 38, 473 (1997).

${ }^{3}$ J. Heinrich, R. Busch, F. Müller, S. Grandthyll, and S. Hüfner, Appl. Phys. Lett. 100, 071909 (2012).

${ }^{4}$ Wah Chang Allegheny Technologies (USA), Technical data sheet Zircadyne 702 @ $/ 705$ ( (2012).

${ }^{5}$ J. Heinrich, R. Busch, and B. Nonnenmacher, Intermetallics 25, 1 (2012).

${ }^{6}$ Wah Chang Allegheny Technologies (USA), Technical Data Sheet Zirconium Crystal Bar (2012).

${ }^{7}$ T. Kordel, D. Holland-Moritz, F. Yang, J. Peters, T. Unruh, T. Hansen, and A. Meyer, Phys. Rev. B 83, 104205 (2011).

${ }^{8}$ P. Heintzmann, F. Yang, S. Schneider, G. Lohöfer, and A. Meyer, Appl. Phys. Lett. 108, 241908 (2016).

${ }^{9}$ J. Brillo, I. Pommrich, and A. Meyer, Phys. Rev. Lett. 107, 165902 (2011).

${ }^{10}$ H. Yoo, C. Park, S. Jeon, S. Lee, and G. W. Lee, Metrologia 52, 677 (2015).

${ }^{11}$ J. Brillo and I. Egry, Int. J. Thermophys. 24, 1155 (2003).

${ }^{12}$ W. Hembree, Ph.D. thesis, University Saarland, 2016.

${ }^{13}$ A. K. Gangopadhyay, C. E. Pueblo, R. Dai, M. L. Johnson, R. Ashcraft, D. Van Hoesen, M. Sellers, and K. F. Kelton, J. Chem. Phys. 146, 154506 (2017).

${ }^{14}$ I. Egry, G. Lohöfer, I. Seyhan, S. Schneider, and B. Feuerbacher, Appl. Phys. Lett. 73, 462 (1998).

${ }^{15}$ C. Way, P. Wadhwa, and R. Busch, Acta Mater. 55, 2977 (2007).

${ }^{16}$ J. Schroers, K. Samwer, F. Szuecs, and W. L. Johnson, J. Mater. Res. 15, 1617 (2000).

${ }^{17}$ Z. Evenson, T. Schmitt, M. Nicola, I. Gallino, and R. Busch, Acta Mater. 60, 4712-4719 (2012).

${ }^{18}$ S. Wei, F. Yang, J. Bednarcik, I. Kaban, O. Shuleshova, A. Meyer, and R. Busch, Nat. Commun. 4, 2083 (2013).

${ }^{19}$ D. Herlach, D. Holland-Moritz, and P. Galenko, Metastable Solids from Undercooled Melts (Pergamon, 2011), Vol. 10.

${ }^{20}$ C. T. Liu, M. F. Chisholm, and M. K. Miller, Intermetallics 10, 1105 (2002).

${ }^{21}$ W. L. Johnson, J. H. Na, and M. D. Demetriou, Nat. Commun. 7, 10313 (2016).

${ }^{22}$ See the supplementary information for Ref. 21, https://www.nature.com/ articles/ncomms10313\#supplementary-information.

${ }^{23}$ X. H. Lin and W. L. Johnson, J. Appl. Phys. 78, 6514 (1995).

${ }^{24}$ S. Mukherjee, J. Schroers, Z. Zhou, W. L. Johnson, and W. K. Rhim, Acta Mater. 52, 3689-3695 (2004).

${ }^{25}$ B. Bochtler, O. Gross, and R. Busch, Appl. Phys. Lett. 111, 261902 (2017). 